\title{
Parametrization of C-shocks. Evolution of the sputtering of grains
}

\author{
I. Jiménez-Serra ${ }^{1,2}$, P. Caselli ${ }^{2,3}$, J. Martín-Pintado ${ }^{1}$, and T. W. Hartquist ${ }^{2}$
}

\author{
${ }^{1}$ Departamento de Astrofísica Molecular e Infrarroja, Instituto de Estructura de la Materia (CSIC), C/ Serrano 121, \\ 28006 Madrid, Spain \\ e-mail: izaskun@damir.iem.csic.es \\ 2 School of Physics and Astronomy, University of Leeds LS2 9JT, Leeds, UK \\ 3 INAF - Osservatorio Astrofisico di Arcetri, Largo E. Fermi 5, 50125 Firenze, Italy
}

Received 11 June 2007 / Accepted 30 January 2008

\section{ABSTRACT}

\begin{abstract}
Context. The detection of narrow $\mathrm{SiO}$ line emission toward the young shocks of the L1448-mm outflow has been interpreted as a signature of the magnetic precursor of $\mathrm{C}$-shocks. In contrast with the low $\mathrm{SiO}$ abundances $\left(\leq 10^{-12}\right)$ derived from the ambient gas, the narrow $\mathrm{SiO}$ emission in the precursor component at almost ambient velocities reveals enhanced $\mathrm{SiO}$ abundances of $\sim 10^{-11}$. It has been proposed that this enhancement of the $\mathrm{SiO}$ abundance is produced by the sputtering of the grain mantles at the early stages of $\mathrm{C}$-shocks. However, modelling of the sputtering of grains has usually averaged the $\mathrm{SiO}$ abundances over the dissipation region of C-shocks, which cannot explain the recent observations.

Aims. We model the evolution of the gas-phase abundances of molecules like $\mathrm{SiO}, \mathrm{CH}_{3} \mathrm{OH}$, and $\mathrm{H}_{2} \mathrm{O}$, produced by the sputtering of the grain mantles and cores as the shock propagates through the ambient gas. We consider different initial gas densities and shock velocities.

Methods. We propose a parametric model to describe the physical structure of C-shocks as a function of time. Using the known sputtering yields for water mantles (with other minor constituents like silicon and $\mathrm{CH}_{3} \mathrm{OH}$ ) and olivine cores by collisions with $\mathrm{H}_{2}$, $\mathrm{He}, \mathrm{C}, \mathrm{O}, \mathrm{Si}, \mathrm{Fe}$, and $\mathrm{CO}$, we follow the evolution of the abundances of silicon, $\mathrm{CH}_{3} \mathrm{OH}$, and $\mathrm{H}_{2} \mathrm{O}$ ejected from grains along the evolution of the shock.

Results. The evolution of the abundances of the sputtered silicon, $\mathrm{CH}_{3} \mathrm{OH}$, and $\mathrm{H}_{2} \mathrm{O}$ shows that $\mathrm{CO}$ seems to be the most efficient sputtering agent in low-velocity shocks. The velocity threshold for the sputtering of silicon from the grain mantles is appreciably reduced (by $5-10 \mathrm{~km} \mathrm{~s}^{-1}$ ) by $\mathrm{CO}$ compared to other models. The sputtering by $\mathrm{CO}$ can generate $\mathrm{SiO}$ abundances of $\sim 10^{-11}$ at the early stages of low-velocity shocks, consistent with those observed in the magnetic precursor component of L1448-mm. Our model satisfactorily reproduces the progressive enhancement of $\mathrm{SiO}, \mathrm{CH}_{3} \mathrm{OH}$, and $\mathrm{H}_{2} \mathrm{O}$ observed in this outflow, suggesting that this enhancement may be due to the propagation of two shocks with $v_{\mathrm{s}}=30 \mathrm{~km} \mathrm{~s}^{-1}$ and $v_{\mathrm{s}}=60 \mathrm{~km} \mathrm{~s}^{-1}$ coexisting within the same region. Conclusions. Our simple model can be used to estimate the time-dependent evolution of the abundances of molecular shock tracers like $\mathrm{SiO}, \mathrm{CH}_{3} \mathrm{OH}, \mathrm{H}_{2} \mathrm{O}$, or $\mathrm{NH}_{3}$ in very young molecular outflows.
\end{abstract}

Key words. ISM: clouds - shock waves - ISM: jets and outflows - ISM: dust, extinction

\section{Introduction}

In young molecular outflows, it is expected that changes in the molecular emission could be observed thanks to the propagation of shocks into the ambient material. So far, the L1448-mm outflow is the only object where time variability of the $\mathrm{SiO}$ emission in the high-velocity jet has been detected, indicating the presence of very young shocks (Girart \& Acord 2001).

It is well known that silicon is heavily depleted onto the grain mantles and grain cores in the quiescent gas of molecular dark clouds like TMC-1, L183, and L1448 (SiO abundance of $\leq 10^{-12}$; Ziurys et al. 1989; Martín-Pintado et al. 1992; Requena-Torres et al. 2007). The detection of large $\mathrm{SiO}$ abundances in regions with outflow activity is therefore a clear indicator of the destruction of dust grains by the interaction of magnetohydrodynamic (MHD) shock waves (or C-shocks) with the ambient gas (Martín-Pintado et al. 1992; Flower et al. 1996; Caselli et al. 1997).

The typical $\mathrm{SiO}$ abundances measured in the highvelocity gas of young molecular outflows such as L1448-mm (Martín-Pintado et al. 1992) are $\geq 10^{-6}$, which implies an enhancement by more than 6 orders of magnitude with respect to the $\mathrm{SiO}$ abundances measured in the quiescent gas. It has been proposed that the detection of very narrow $\mathrm{SiO}$ emission at almost ambient velocities toward this outflow is produced by the magnetic precursor of C-shocks (Jiménez-Serra et al. 2004). The $\mathrm{SiO}$ abundance of $\sim 10^{-11}$ for this narrow emission clearly contrasts with the large $\mathrm{SiO}$ enhancement found in the high-velocity postshock gas and with the much lower $\mathrm{SiO}$ abundance of the quiescent material.

Toward the young shocks of the L1448-mm outflow, Jiménez-Serra et al. (2005) also report an evolutionary trend in the $\mathrm{SiO}$ and $\mathrm{CH}_{3} \mathrm{OH}$ abundances (methanol is the most abundant molecule after $\mathrm{H}_{2} \mathrm{O}$ in the grain mantles; Tielens \& Allamandola 1987) to be enhanced from the ambient gas to the moderatevelocity component, as if the grain mantles would have been progressively eroded by the recent interaction of low-velocity shocks.

Modelling of C-shocks that only includes the sputtering of grain cores shows that an appreciable fraction of silicon material starts to be ejected from grains for $v_{\mathrm{s}} \geq 25-30 \mathrm{~km} \mathrm{~s}^{-1}$ (Flower et al. 1996; Caselli et al. 1997; May et al. 2000). Although these models predict $\mathrm{SiO}$ abundances that are consistent with those observed in the postshock gas $\left(\sim 10^{-8}-10^{-7}\right)$, the sputtering 
of $\mathrm{SiO}$ from the cores cannot reproduce the lower $\mathrm{SiO}$ abundances of $\sim 10^{-11}$ found in the narrow precursor component of L1448-mm.

Calculations of the sputtering yield of silicon by heavy atoms like $\mathrm{C}, \mathrm{O}, \mathrm{Si}$, and $\mathrm{Fe}$ on $\mathrm{SiO}_{2}$ and olivine $\left(\mathrm{MgFeSiO}_{4}\right)$ cores show that, despite the low relative abundances of these species with respect to $\mathrm{H}_{2}$ and $\mathrm{He}$ in dark clouds, these heavy particles can dominate the sputtering of grains at low shock velocities (Field et al. 1997; May et al. 2000). Furthermore, abundant molecules like $\mathrm{CO}$ could also play an important role in the sputtering of dust grains, since these species can sputter like atoms of equivalent mass for low impact velocities (May et al. 2000). Considering that silicon could be a minor constituent of the mantles, their sputtering by these heavy species in low-velocity shocks could efficiently erode them, generating the $\mathrm{SiO}$ abundances observed for the narrow $\mathrm{SiO}$ line emission in L1448-mm. Up to now, the evolution of the sputtering of grains has not been studied in detail. The questions of which species are the most efficient sputtering agents and which timescales are needed to eject most of the silicon material from grains still remain uncertain.

In this paper, we present a parametric model of $\mathrm{C}$-shocks to describe in detail the time dependent evolution of the molecular abundances sputtered from grains in low and high-velocity shocks. This approximation constitutes a powerful tool for interpreting the molecular abundances measured in young molecular outflows. In addition to $\mathrm{H}_{2}$ and $\mathrm{He}$, heavy atoms and molecules have been also considered as sputtering agents. In Sect. 2, we present the approximations used to describe the steady state profile of the physical structure of C-shocks. In Sect. 3, we show the procedure for determining the sputtering of the grain mantles and the grain cores. In Sect. 4, we present the results of the sputtering of silicon from grains for several initial gas densities and shock velocities. In Sects. 5 and 6, we compare the sputtered $\mathrm{SiO}, \mathrm{CH}_{3} \mathrm{OH}$, and $\mathrm{H}_{2} \mathrm{O}$ abundances with those measured in the L1448-mm outflow. The conclusions are finally summarised in Sect. 7.

\section{The C-shock structure in the preshock frame}

We consider a plane-parallel C-shock that propagates through the quiescent gas with velocity $v_{\mathrm{s}}$. As a first approximation, we have assumed steady state profiles for the evolution of the physical parameters in the shock. The validity of this approximation, versus more recent time-dependent modelling of the physical structure of C-shocks, will be discussed in detail in Sect. 4.1.

The initial $\mathrm{H}_{2}$ density and temperature of the ambient cloud are $n_{0}$ and $T_{0}$, respectively. Since one of the aims of this work is to directly compare our results with observations toward the young L1448-mm outflow, it is convenient to consider that the velocities of the ion and neutral fluids, $v_{\mathrm{i}}$ and $v_{\mathrm{n}}$, are in the frame co-moving with the preshock gas. These velocities are approximated by

$v_{\mathrm{n}, \mathrm{i}}=\left(v_{\mathrm{s}}-v_{0}\right)-\frac{\left(v_{\mathrm{s}}-v_{0}\right)}{\cosh \left[\left(z-z_{0}\right) / z_{\mathrm{n}, \mathrm{i}}\right]}$

where $z$ is the spatial coordinate and the $z_{\mathrm{n}} / z_{\mathrm{i}}$ ratio governs the strength of the velocity decoupling between the ion and neutral fluids. The variable $z_{0}$ corresponds to the distance at which these fluids start to decouple (see Sect.4.1 for details on how to estimate these parameters). An additional velocity, $v_{0}$, also needs to be considered in the equations for $v_{\mathrm{n}}$ and $v_{\mathrm{i}}$ to avoid infinite compression of the far downstream gas (see Eq. (3) below). The variable $v_{0}$ depends on the shock parameters and is defined as the final downstream velocity of the ion and neutral fluids in the shock frame (in the preshock frame, this final velocity would be $v_{\mathrm{s}}-v_{0}$; see Sect. 4.1). As shown in Appendix A, this velocity is tightly linked to the shock and Alfvén velocities, $v_{\mathrm{s}}$ and $v_{\mathrm{A}}$, through shock jump conditions.

The ion-neutral drift speed $v_{\mathrm{d}}$ is $v_{\mathrm{d}}=\left|v_{\mathrm{n}}-v_{\mathrm{i}}\right|$, and the neutral fluid flow time is calculated as (see Eqs. (A.7) and (B.10))

$t=\int \frac{\mathrm{d} z}{v_{\mathrm{s}}-v_{\mathrm{n}}}$

From the principle of mass conservation, the neutral density, $n_{\mathrm{n}}$, is given by

$n_{\mathrm{n}}=\frac{n_{0} v_{\mathrm{s}}}{v_{\mathrm{s}}-v_{\mathrm{n}}}$

The temperature of the neutral fluid, $T_{\mathrm{n}}$, is approximated by a Planck-like function as

$T_{\mathrm{n}}=T_{0}+\frac{\left[a_{T}\left(z-z_{0}\right)\right]^{b_{\mathrm{T}}}}{\exp \left[\left(z-z_{0}\right) / z_{\mathrm{T}}\right]-1}$

where $b_{\mathrm{T}}$ is an integer, and $a_{\mathrm{T}}$ and $z_{\mathrm{T}}$ are related to the maximum value of $T_{\mathrm{n}}\left(T_{\mathrm{n}, \max }\right)$ and the distance $z_{\mathrm{n}, \max }$ at which $T_{\mathrm{n}}$ reaches its maximum value. The temperature of the ion fluid is calculated by using $T_{\mathrm{i}}=T_{\mathrm{n}}+\left(\frac{m v_{\mathrm{d}}^{2}}{3 k}\right)$.

The comparison of the model predictions with observations (Sects. 5 and 6) requires consideration of the radial velocity of the preshock gas (ambient cloud gas) relative to the observer, $v_{\mathrm{cl}}$. This velocity, the radial velocity of the emission measured by the observer, $v_{\mathrm{LSR}}$, and the velocity of the neutral fluid as measured in the frame of the ambient medium, $v_{\mathrm{n}}$, are related by

$v_{\mathrm{LSR}}=v_{\mathrm{cl}}+v_{\mathrm{n}}$.

In Appendix A, we also give the equations for $v_{\mathrm{n}}, v_{\mathrm{i}}$, and $n_{\mathrm{n}}$ within the frame of the shock (see Eqs. (A.1) and (A.5)) that will be used in Sect. 4.1 to validate this parametric approximation.

\section{Sputtering of grains}

In this section, we describe the sputtering of grains produced by collisions with $\mathrm{H}_{2}$ and $\mathrm{He}$ and other heavy atomic and molecular species such as $\mathrm{C}, \mathrm{O}, \mathrm{Si}, \mathrm{Fe}$, and $\mathrm{CO}$ (see Appendix B for the full explanation of the method). Although we consider that most silicon is locked into the olivine grain cores, we assume that a small fraction of this element is also present within the icy water mantles $\left(q_{\mathrm{m}}=1.4 \times 10^{-4}\right.$; see below). The molecule $\mathrm{CH}_{3} \mathrm{OH}$ has been also considered as another constituent of the icy mantles.

\subsection{Sputtering of the grain mantles}

To study the sputtering of the grain mantles, we followed the procedure described by Caselli et al. (1997). The sputtering rate per unit volume and grain (Eq. (B.1) in Appendix B) has been derived by averaging the sputtering yield at low energies (Eq. (B.2)) over a velocity-shifted Maxwellian distribution characterised by $T_{\mathrm{n}}$ and $v_{\mathrm{d}}$. The surface binding energy $U_{0}$ of the water mantles is $0.53 \mathrm{eV}$ (Tielens et al. 1994). The projectile masses $m_{\mathrm{p}}$ are 2, 4, 12, 16, 28, 56, and $28 \mathrm{amu}$ for $\mathrm{H}_{2}, \mathrm{He}, \mathrm{C}$, $\mathrm{O}, \mathrm{Si}, \mathrm{Fe}$, and $\mathrm{CO}$, respectively. The target mass $M_{\mathrm{t}}$ is considered to be $18 \mathrm{amu}$, which corresponds to the molecular mass of $\mathrm{H}_{2} \mathrm{O}$. The initial fractional abundances of $\mathrm{He}, \mathrm{C}, \mathrm{O}, \mathrm{Si}, \mathrm{Fe}$, and $\mathrm{CO}$, relative to atomic hydrogen, are shown in Table 1 . We 
Table 1. Initial gas phase abundances of $\mathrm{He}, \mathrm{C}, \mathrm{O}, \mathrm{Si}, \mathrm{Fe}$, and $\mathrm{CO}$.

\begin{tabular}{cc}
\hline \hline $\begin{array}{c}\text { Element/ } \\
\text { molecule }\end{array}$ & $\begin{array}{c}\text { Abundance }^{a} \\
{[n(\mathrm{X}) / n(\mathrm{H})]}\end{array}$ \\
\hline $\mathrm{He}$ & 0.1 \\
$\mathrm{C}$ & $7.1 \times 10^{-9}$ \\
$\mathrm{O}$ & $1.8 \times 10^{-4}$ \\
$\mathrm{Si}$ & $8.0 \times 10^{-9}$ \\
$\mathrm{Fe}$ & $3.0 \times 10^{-9}$ \\
$\mathrm{CO}$ & $1.5 \times 10^{-4}$ \\
\hline
\end{tabular}

${ }^{a}$ From the low metal case for dense cloud chemistry (Prasad \& Huntress 1982; Herbst \& Leung 1989; Graedel et al. 1982).

Table 2. Fractional abundances of $\mathrm{H}_{2} \mathrm{O}, \mathrm{CH}_{3} \mathrm{OH}$, and $\mathrm{Si} / \mathrm{SiO}$ assumed for the icy mantles and the grain cores.

\begin{tabular}{ccc}
\hline \hline Species & \multicolumn{2}{c}{ Abundances $[\chi]$} \\
& Mantles & Cores \\
\hline $\mathrm{H}_{2} \mathrm{O}$ & $7.25 \times 10^{-5 a}$ & $\ldots$ \\
$\mathrm{CH}_{3} \mathrm{OH}$ & $\sim 10^{-6 b}$ & $\ldots$ \\
$\mathrm{Si} / \mathrm{SiO}$ & $\sim 10^{-8 b}$ & $\sim 3.6 \times 10^{-5 c}$ \\
\hline
\end{tabular}

${ }^{a}$ From Whittet \& Duley (1991). ${ }^{b}$ From Jiménez-Serra et al. (2005).

${ }^{c}$ From Anders \& Grevesse (1989) and Snow \& Witt (1996).

assume that these abundances remain constant throughout the dissipation region of the shock. The volume density of grains, $n_{\mathrm{g}}$, is derived by considering a gas-to-dust mass ratio of $\sim 100$, a constant grain radius of $0.1 \mu \mathrm{m}$ and a density of the grain core material of $3.5 \mathrm{~g} \mathrm{~cm}^{-3}$ (most of the volume of a grain is filled by the silicate core; Caselli et al. 1997). The total sputtering rate for $\mathrm{H}_{2} \mathrm{O}, \mathrm{CH}_{3} \mathrm{OH}$, and silicon are calculated with Eqs. (B.4)-(B.6). The total volume densities of these species are finally estimated with Eqs. (B.7)-(B.9). Since the amount of material contained within the grain mantles is limited, we assume that the maximum abundances of silicon and $\mathrm{CH}_{3} \mathrm{OH}$ ejected from the mantles are those of $\mathrm{SiO}$ and $\mathrm{CH}_{3} \mathrm{OH}$ measured in the low-velocity gas of the L1448-mm outflow $\left(\sim 10^{-8}\right.$ and $\sim 10^{-6}$, respectively; see Table 2 and Jiménez-Serra et al. 2005). From the observations and assuming a $\mathrm{H}_{2} \mathrm{O}$ abundance of $\sim 7.25 \times 10^{-5}$ (Table 2 and Whittet \& Duley 1991), we can derive the fraction of silicon, $q_{\mathrm{m}}$, and $\mathrm{CH}_{3} \mathrm{OH}, r_{\mathrm{m}}$, present within the water mantles as $q_{\mathrm{m}}=\chi(\mathrm{SiO}) / \chi\left(\mathrm{H}_{2} \mathrm{O}\right)=1.4 \times 10^{-4}$ and $r_{\mathrm{m}}=\chi\left(\mathrm{CH}_{3} \mathrm{OH}\right) / \chi\left(\mathrm{H}_{2} \mathrm{O}\right)=1.4 \times 10^{-2}$. Although $q_{\mathrm{m}}$ and $r_{\mathrm{m}}$ constitute free parameters, we have fixed their values for comparison purposes with the L1448-mm outflow (Sects. 5 and 6).

\subsection{Sputtering of the grain cores}

For the sputtering of the cores, we used different approaches to calculate the sputtering produced by collisions with $\mathrm{H}_{2}$ and by collisions with $\mathrm{He}, \mathrm{C}, \mathrm{O}, \mathrm{Si}, \mathrm{Fe}$, and $\mathrm{CO}$. We assumed that olivine $\left(\mathrm{MgFeSiO}_{4}\right)$ is the main form of silicates in the cores. In the case of $\mathrm{H}_{2}$, we calculated the angle-averaged sputtering yield as in Sect. 3.1 (see Eq. (B.2) in Appendix B), but considering a surface binding energy $U_{0}=5.70 \mathrm{eV}$ for the silicate cores (Tielens et al. 1994). For the sputtering agents He, C, O, $\mathrm{Si}$, and $\mathrm{Fe}$, we used the sputtering yields for olivine calculated by May et al. (2000, see Eq. (B.3)). Since CO has the same projectile mass as $\mathrm{Si}$, we assumed that the sputtering yield of $\mathrm{CO}$ is similar to that of Si (Field et al. 1997; May et al. 2000). The sputtering threshold energies $E_{\text {th }}$ used for the projectiles are $73 \mathrm{eV}$ for $\mathrm{He}, 48 \mathrm{eV}$ for $\mathrm{C}$, and $47 \mathrm{eV}$ for $\mathrm{O}, \mathrm{Si}, \mathrm{Fe}$, and $\mathrm{CO}$ (Table 4 of May et al. 2000). To take the projection effects into account in the production of silicon within the shock, we included the factor $1 / \cos ^{2} \theta$ in the impact energy $E_{\mathrm{p}}$ of the colliding particle (Eq. (B.3)), where $\theta$ is the inclination angle of the outflow with respect to the line of sight. For the L1448-mm outflow (see Sects. 5 and 6), $\theta$ is $\sim 70^{\circ}$ (Girart \& Acord 2001). As for the mantles, the total sputtering rate for silicon is determined by Eq. (B.6), and Eq. (B.9) calculates the volume density of silicon ejected from the grain cores. In the case of $\mathrm{H}_{2}$, we also need to consider that the probability for a silicon atom to be injected into the gas phase from an olivine molecule, as opposed to an $\mathrm{Mg}, \mathrm{Fe}$, or $\mathrm{O}$ atom, $q_{\mathrm{c}}$ is 0.2 (Caselli et al. 1997). However, for the rest of the colliding particles, we assumed that $q_{\mathrm{c}}=1$ since this probability has already been taken into account in the calculations of the sputtering yields of May et al. (2000). Practically all silicon $(\sim 99.97 \%)$ is locked into the grain cores with an abundance of $\sim 3.6 \times 10^{-5}$ (see Table 2; Anders \& Grevesse 1989; Snow \& Witt 1996).

\section{Results}

\subsection{Validation of the approximation of the physical structure of the $\mathrm{C}$-shock}

To validate the parametric approximation of the C-shock physical structure of Sect. 2, in Fig. 1, we show the comparison between the C-shock profile, calculated as in the recent MHD models of Flower \& Pineau des Forêts (2003), and the profiles of $v_{\mathrm{n}}, v_{\mathrm{i}}, \Delta v, T_{\mathrm{n}}$, and $T_{\mathrm{i}}$ derived in the shock frame through our approach (see Appendix A for details). In Fig. 2, we directly compare our results with the MHD shock structure obtained by Kaufman \& Neufeld (1996). The values of $z_{\mathrm{n}}, z_{\mathrm{i}}, z_{\mathrm{T}}, z_{0}$, and $a_{\mathrm{T}}$ chosen to reproduce these shock profiles (see below for the estimation of the input parameters), and the values of the magnetic field, $B_{0}$, fractional ionization, $\chi_{\mathrm{e}}$, shock length scale, $\Delta$, and maximum temperature of the neutral fluid, $T_{\mathrm{n}, \max }$, are shown in Table 3.

From Fig. 1, it is clear that, although some differences do exist between the approximation and the MHD shock modelling at moderate preshock densities (Flower, private communication), the general behaviour of $v_{\mathrm{n}}, v_{\mathrm{i}}, \Delta v, T_{\mathrm{n}}$, and $T_{\mathrm{i}}$, qualitatively mimics the physical structure of C-shocks. In particular, the velocity decoupling between the ion and neutral fluids in the magnetic precursor, and the initial delay in the switch on of the neutral heating at this stage are well reproduced by our approximation. Note that the agreement between the profiles of $v_{\mathrm{n}}$ and $v_{\mathrm{i}}$ is excellent, giving a reliable prediction of $\Delta v$, which is a key parameter in the sputtering yield calculation (see Appendix B). To fit the delay of the heating of the neutrals at the magnetic precursor stage, we need to impose $b_{\mathrm{T}} \geq 6$.

For higher preshock densities, Fig. 2 shows that the ionneutral velocity decoupling in the magnetic precursor is not as accurately reproduced as in Fig. 1 for the moderate density case (Flower \& Pineau des Forêts 2003). We should mention, however, that the MHD treatment of C-shocks for high density regions, is still rather simplistic and, therefore, uncertain (see Pilipp et al. 1990; Pilipp \& Hartquist 1994; Falle 2003; Chapman $\&$ Wardle 2006). Since the existing perpendicular shock models may not start having problems until the preshock density exceeds $10^{6} \mathrm{~cm}^{-3}$ (see e.g. Pilipp et al. 1990), in the following we restrict our study to the moderate density case (from $10^{4}$ to $10^{6} \mathrm{~cm}^{-3}$ ).

In Table 4, we summarise the different values of $z_{\mathrm{n}}, z_{\mathrm{i}}, z_{\mathrm{T}}$, and $a_{\mathrm{T}}$, chosen to reproduce the physical structure of a sample of C-shocks with velocities of $10 \leq v_{\mathrm{s}} \leq 40 \mathrm{~km} \mathrm{~s}^{-1}$ and initial $\mathrm{H}_{2}$ densities of $10^{4} \leq n_{0} \leq 10^{6} \mathrm{~cm}^{-3}$. Both $z_{\mathrm{n}}$ and $z_{\mathrm{i}}$ have been 

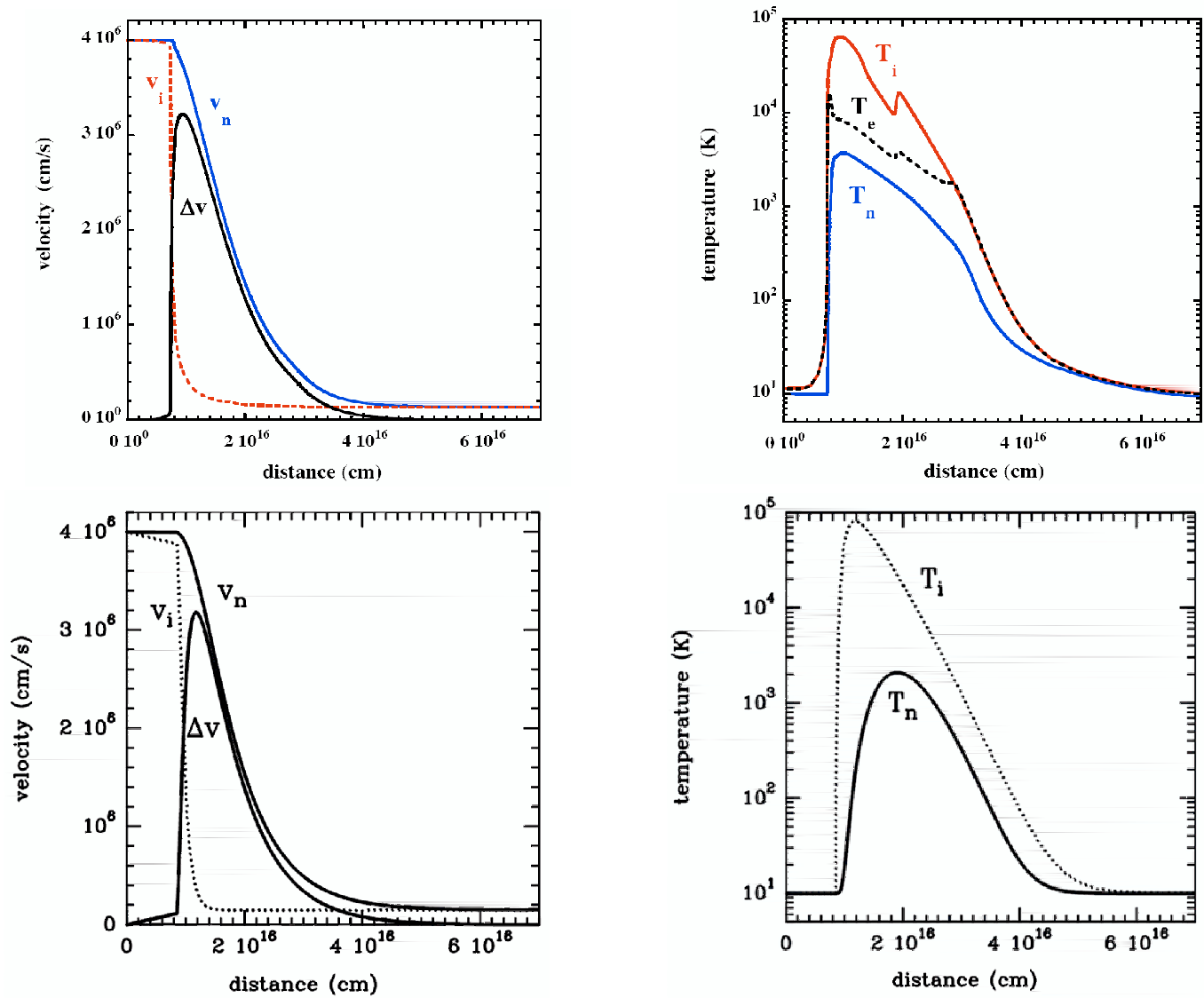

Fig. 1. Comparison between our approximation of $v_{\mathrm{n}}, v_{\mathrm{i}}, \Delta v, T_{\mathrm{n}}$, and $T_{\mathrm{i}}$ (lower panels), and the MHD shock structure calculated as in Flower \& Pineau des Forêts (2003, upper panels) for a shock with $v_{\mathrm{s}}=40 \mathrm{~km} \mathrm{~s}^{-1}, n_{0}=10^{4} \mathrm{~cm}^{-3}$ and $B_{0}=100 \mu \mathrm{G}$ (Flower, private communication). Shock parameters are given in Table 3 and velocities are in the shock frame (see Appendix A for the definition of $v_{\mathrm{n}}$ and $v_{\mathrm{i}}$ ).

estimated by considering that $v_{\mathrm{n}}$ is $0.999 v_{\mathrm{s}}$ at $\Delta$ and by assuming a $z_{\mathrm{n}} / z_{\mathrm{i}}$ ratio of $\sim 9 / 2$. We note that slightly higher (factor of 1.5 ) $z_{\mathrm{n}} / z_{\mathrm{i}}$ ratios are required to reproduce the results of Flower \& Pineau des Forêts (2003) with a new treatment of the coupling between the neutral and the charged fluids (Flower, private communication). However, for consistency, we will hereafter use the $z_{\mathrm{n}} / z_{\mathrm{i}}$ ratio of $\sim 9 / 2$, since it reproduces the results of Flower et al. (1996) and Draine et al. (1983), for which Dopita \& Sutherland (2003) accordingly give an estimate of the shock length scale, $\Delta$. In either case, the results obtained with both values of $z_{\mathrm{n}} / z_{\mathrm{i}}$ do not differ by more than $15 \%$.

The parameters $a_{\mathrm{T}}$ and $z_{\mathrm{T}}$ were derived by assuming that $b_{\mathrm{T}}=6$ and $z_{0}=0 \mathrm{~cm}$. The magnetic field, $B_{0}$, and fractional ionization of the gas, $\chi_{\mathrm{e}}$, were calculated as in Draine et al. (1983), and the shock length scale, $\Delta$, as in Dopita \& Sutherland (2003). The estimated Alfvén velocity is $v_{\mathrm{A}}=2.18 \mathrm{~km} \mathrm{~s}^{-1}$, and $v_{0}$ typically ranges from 3.1 to $4.7 \mathrm{~km} \mathrm{~s}^{-1}$ for the cases considered in Table 4 (see Appendix A for the calculation of $v_{\mathrm{A}}$ and $v_{0}$ ). We note that the shock length scales derived for each initial $\mathrm{H}_{2}$ density of Table 4, are within the same order of magnitude as the ion-neutral coupling lengths determined by Kaufman \& Neufeld (1996, see Fig. 1 in this work). The maximum temperature of the neutral fluid, $T_{\mathrm{n} \text {,max }}$, was estimated from the results of Draine et al. (1983).

In Fig. 3, we show a representative profile of a $\mathrm{C}$-shock with $v_{\mathrm{s}}=40 \mathrm{~km} \mathrm{~s}^{-1}, n_{0}=10^{5} \mathrm{~cm}^{-3}$, and $T_{0}=10 \mathrm{~K}$. As expected in the frame co-moving with the preshock gas, the ion and neutral

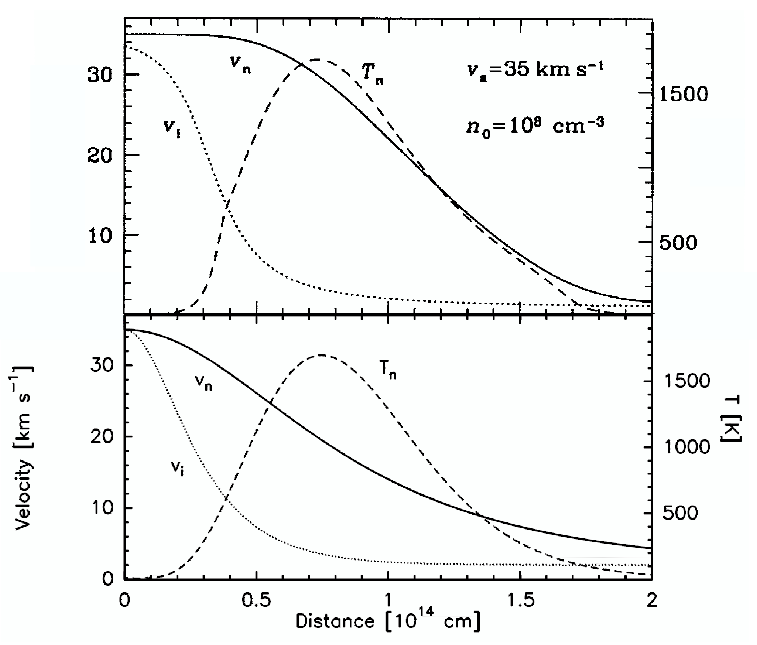

Fig. 2. Comparison between the profiles of $v_{\mathrm{n}}, v_{\mathrm{i}}$ and $T_{\mathrm{n}}$ derived with our approximation (lower panel) and the MHD shock structure obtained by Kaufman \& Neufeld (1996) for a shock with $v_{\mathrm{s}}=35 \mathrm{~km} \mathrm{~s}^{-1}$ and $n_{0}=10^{8} \mathrm{~km} \mathrm{~s}^{-1}$ (upper panel). Parameters are given in Table 3 and velocities are in the shock frame.

fluids are at rest at the beginning of the shock, and their final velocities in the far downstream gas are of $\sim v_{\mathrm{s}}-v_{0}$ (see Draine et al. 1983). The initialdelay of the heating of the neutrals gives 
Table 3. Input parameters for the C-shock profiles shown in Figs. 1 and 2.

\begin{tabular}{ccccccccccc}
\hline \hline $\begin{array}{c}v_{\mathrm{s}} \\
\left(\mathrm{km} \mathrm{s}^{-1}\right)\end{array}$ & $\begin{array}{c}n\left(\mathrm{H}_{2}\right) \\
\left(\mathrm{cm}^{-3}\right)\end{array}$ & $\begin{array}{c}B_{0} \\
(m \mathrm{G})\end{array}$ & $\chi \mathrm{e}$ & $\begin{array}{c}z_{\mathrm{n}} \\
(\mathrm{cm})\end{array}$ & $\begin{array}{c}z_{\mathrm{i}} \\
(\mathrm{cm})\end{array}$ & $\begin{array}{c}z_{\mathrm{T}} \\
(\mathrm{cm})\end{array}$ & $\begin{array}{c}z_{0} \\
(\mathrm{~cm})\end{array}$ & $\begin{array}{c}a_{\mathrm{T}} \\
\left(\mathrm{K}^{1 / 6} \mathrm{~cm}^{-1}\right)\end{array}$ & $\begin{array}{c}\Delta \\
(\mathrm{cm})\end{array}$ & $\begin{array}{c}T_{\mathrm{n}, \max } \\
(\mathrm{K})\end{array}$ \\
\hline 40 & $10^{4}$ & 0.14 & $7 \times 10^{-8}$ & $7.0 \times 10^{15}$ & $1.0 \times 10^{15}$ & $1.8 \times 10^{15}$ & $8.0 \times 10^{15}$ & $9.0 \times 10^{-16}$ & $5 \times 10^{16}$ & 2000 \\
35 & $10^{8}$ & 14 & $7 \times 10^{-10}$ & $6.0 \times 10^{13}$ & $2.0 \times 10^{13}$ & $1.3 \times 10^{13}$ & 0.0 & $1.3 \times 10^{-13}$ & $2 \times 10^{14}$ & 1700 \\
\hline
\end{tabular}

Table 4. Input parameters for a sample of C-shocks.

\begin{tabular}{ccccccccccc}
\hline \hline $\begin{array}{c}v_{\mathrm{s}} \\
\left(\mathrm{km} \mathrm{s}^{-1}\right)\end{array}$ & $\begin{array}{c}v_{0}^{a} \\
\left(\mathrm{~km} \mathrm{~s}^{-1}\right)\end{array}$ & $\begin{array}{c}n\left(\mathrm{H}_{2}\right) \\
\left(\mathrm{cm}^{-3}\right)\end{array}$ & $\begin{array}{c}B_{0}{ }^{b} \\
(\mu \mathrm{G})\end{array}$ & $\chi_{\mathrm{e}}^{b}$ & $\begin{array}{c}z_{\mathrm{n}} \\
(\mathrm{cm})\end{array}$ & $\begin{array}{c}z_{\mathrm{i}} \\
(\mathrm{cm})\end{array}$ & $\begin{array}{c}z_{\mathrm{T}}{ }^{2} \\
(\mathrm{~cm})\end{array}$ & $\begin{array}{c}a_{\mathrm{T}}{ }^{c} \\
\left(\mathrm{~K}^{1 / 6} \mathrm{~cm}^{-1}\right)\end{array}$ & $\begin{array}{c}\Delta^{d} \\
(\mathrm{pc})\end{array}$ & $\begin{array}{c}T_{\mathrm{n}, \max }{ }^{e} \\
(\mathrm{~K})\end{array}$ \\
\hline 20 & 3.8 & $10^{4}$ & 140 & $7 \times 10^{-8}$ & $1.4 \times 10^{16}$ & $3.2 \times 10^{15}$ & $5.0 \times 10^{15}$ & $2.9 \times 10^{-16}$ & 0.024 & 900 \\
40 & 4.7 & $10^{4}$ & 140 & $7 \times 10^{-8}$ & $2.8 \times 10^{16}$ & $6.2 \times 10^{15}$ & $1.1 \times 10^{16}$ & $1.5 \times 10^{-16}$ & 0.048 & 2200 \\
10 & 3.1 & $10^{5}$ & 450 & $2 \times 10^{-8}$ & $7.7 \times 10^{14}$ & $1.7 \times 10^{14}$ & $2.0 \times 10^{14}$ & $5.8 \times 10^{-15}$ & 0.0012 & 300 \\
20 & 3.8 & $10^{5}$ & 450 & $2 \times 10^{-8}$ & $1.4 \times 10^{15}$ & $3.2 \times 10^{14}$ & $5.0 \times 10^{14}$ & $2.8 \times 10^{-15}$ & 0.0024 & 800 \\
30 & 4.3 & $10^{5}$ & 450 & $2 \times 10^{-8}$ & $2.1 \times 10^{15}$ & $4.7 \times 10^{14}$ & $8.0 \times 10^{14}$ & $2.0 \times 10^{-15}$ & 0.0036 & 2000 \\
40 & 4.7 & $10^{5}$ & 450 & $2 \times 10^{-8}$ & $2.8 \times 10^{15}$ & $6.2 \times 10^{14}$ & $1.1 \times 10^{15}$ & $1.6 \times 10^{-15}$ & 0.0048 & 4000 \\
20 & 3.8 & $10^{6}$ & 1400 & $7 \times 10^{-9}$ & $1.4 \times 10^{14}$ & $3.2 \times 10^{13}$ & $5.0 \times 10^{13}$ & $2.8 \times 10^{-14}$ & $2.4 \times 10^{-4}$ & 800 \\
40 & 4.7 & $10^{6}$ & 1400 & $7 \times 10^{-9}$ & $2.8 \times 10^{14}$ & $6.2 \times 10^{13}$ & $1.1 \times 10^{14}$ & $1.6 \times 10^{-14}$ & $4.8 \times 10^{-4}$ & 4000 \\
\hline
\end{tabular}

${ }^{a}$ Calculated with Eq. (A.4) (see Appendix A) and assuming $v_{\mathrm{A}}=2.18 \mathrm{~km} \mathrm{~s}^{-1} .{ }^{b}$ Estimated using Eqs. (62) and (63) of Draine et al. (1983).

${ }^{c}$ Calculated considering that $b_{\mathrm{T}}=6$ and $z_{0}=0 \mathrm{~cm} .{ }^{d}$ Derived as in Dopita \& Sutherland (2003) and assuming that $n_{0, \mathrm{i}} / n_{\mathrm{H}} \sim 10^{-6}$. ${ }^{e}$ Taken from Figs. $8 \mathrm{~b}$ and $9 \mathrm{~b}$ of Draine et al. (1983) for $n_{0}=10^{4} \mathrm{~cm}^{-3}$, and $n_{0}=10^{5}$, and $10^{6} \mathrm{~cm}^{-3}$ respectively.

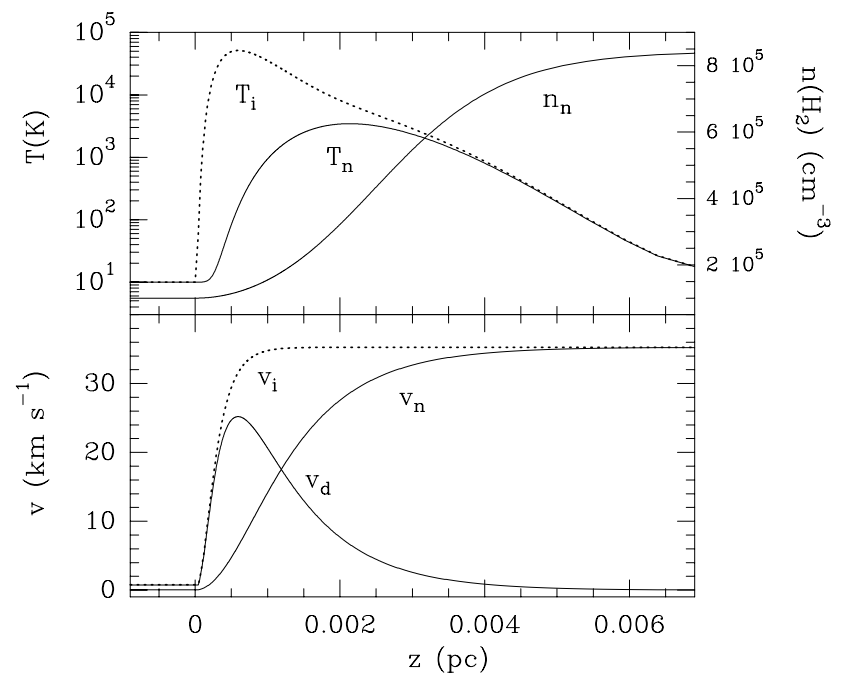

Fig. 3. C-shock physical structure obtained with Eqs. (1), (3), and (4) for $v_{\mathrm{s}}=40 \mathrm{~km} \mathrm{~s}^{-1}, n_{0}=10^{5} \mathrm{~cm}^{-3}$, and $T_{0}=10 \mathrm{~K}$. Velocities are in the frame co-moving with the preshock gas. The magnetic precursor length is of $\Delta z \sim 0.0005-0.001 \mathrm{pc}=1.5-3.0 \times 10^{15} \mathrm{~cm}$.

the magnetic precursor length, which is $\Delta z \sim 0.0005-0.001 \mathrm{pc}$ $\sim 1.5-3.0 \times 10^{15} \mathrm{~cm}$ (Fig. 3). While the maximum value of the temperature of the ions is correlated with the maximum value of $v_{\mathrm{d}}$ (see Fig. 3), the neutrals show their maximum temperature at $v_{\mathrm{n}} \sim 0.85 v_{\mathrm{s}}$ (derived by assuming $\mathrm{H}_{2} \mathrm{O}$ cooling and $\alpha_{\mathrm{c}}=1.5$ in Eq. (18) of Smith \& Brand 1990), which is consistent with the results of Kaufman \& Neufeld (1996, see Fig. 2).

From the recent results of time-dependent shock modelling, one may consider that our assumption of steadiness for the C-shock could not be valid enough to describe the time evolution of the sputtering of grains. These models indeed show that a J-type component is a natural feature in the far downstream gas of the C-shock (near the piston) for timescales of $\leq 10^{3}-10^{4} \mathrm{yr}$, for which the steady state is finally attained (Chièze et al. 1998; Lesaffre et al. 2004). However, as shown in Sect. 4.2, the evolutionary stages relevant to the main injection of the material contained in the icy mantles and in the grain cores are those of the magnetic precursor that, independent of the age of the shock, can be described by the steady state profile of C-shocks (Chièze et al. 1998; Lesaffre et al. 2004).

\subsection{Sputtered silicon abundances: injection and saturation times.}

We now include the evolutionary profiles of $v_{\mathrm{n}}, v_{\mathrm{i}}, T_{\mathrm{n}}, T_{\mathrm{i}}$, and $n_{\mathrm{n}}$ from Sect. 4.1 in the sputtering equations of the Appendix B to calculate the silicon abundances ejected from the mantles and from the cores. Figure 4 shows the silicon abundances ejected from grains as a function of the flow time for several $\mathrm{H}_{2}$ gas densities and shock velocities. The abundances of sputtered silicon hardly change with the initial density of the gas, which clearly agrees with the results of Caselli et al. (1997). However, as expected from the strong dependence of the sputtering rate on the maximum value of $v_{\mathrm{d}}$ (see Eq. (B.1) and Pineau des Forêts et al. 1997), the silicon abundance is drastically enhanced by increasing shock velocities. From Fig. 4, we also note that the timescales are progressively reduced by nearly a factor of 10 as we increase the $\mathrm{H}_{2}$ density from $10^{4}$ to $10^{5}$ and $10^{6} \mathrm{~cm}^{-3}$. This is consistent with the flow time, $t$, being inversely proportional to the density (the cooling timescales roughly vary as $n_{\mathrm{i}}^{-1}$, where $n_{\mathrm{i}}$ is proportional to the density; see Chièze et al. 1998; Lesaffre et al. 2004).

In Fig. 5, we show the products of the sputtering of the mantles and of the cores for an initial density of $10^{5} \mathrm{~cm}^{-3}$ and for shock velocities of $10,20,30$ and $40 \mathrm{~km} \mathrm{~s}^{-1}$. The sputtering of the grain mantles by collisions with $\mathrm{H}_{2}$ and $\mathrm{He}$ (bold lines in Fig. 5) corresponds exactly to what was previously calculated by Caselli et al. (1997). Although the fractional abundance of the heavy species is orders of magnitude smaller than that of $\mathrm{H}_{2}$ and $\mathrm{He}$ (see Sect. 3.1), it is clear that the heavy atoms and $\mathrm{CO}$ sputter the mantles much more efficiently than $\mathrm{H}_{2}$ or He for low shock velocities (Fig. 5).

The high efficiency of these heavy species as sputtering agents is also shown by the injection, $t_{\mathrm{inj}}$, and saturation times, $t_{\text {sat }}$, of Table 5. We define $t_{\text {inj }}$ as the time for which the gas phase silicon abundance, relative to $\mathrm{H}_{2}$, exceeds $10^{-20}$ (i.e. the 


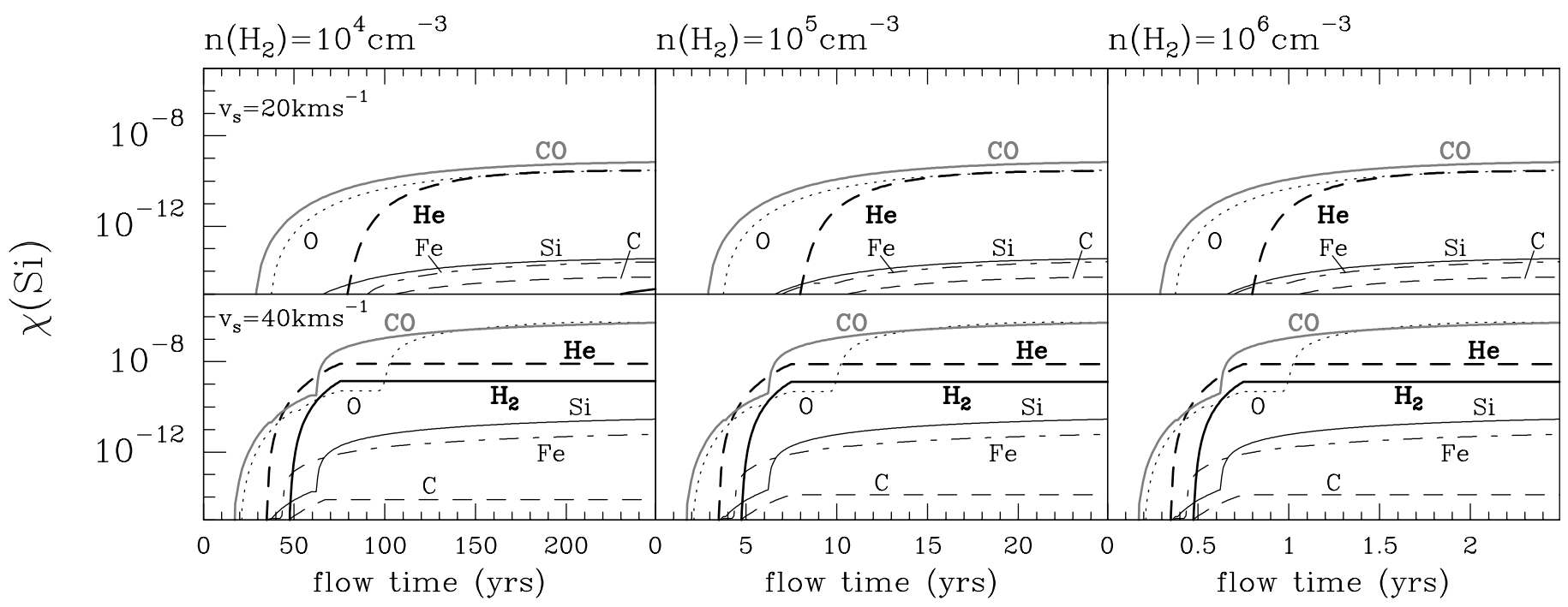

Fig. 4. Evolution of the abundance of elemental silicon ejected from grains by the impact with $\mathrm{H}_{2}, \mathrm{He}, \mathrm{C}, \mathrm{O}, \mathrm{Si}, \mathrm{Fe}$, and $\mathrm{CO}$, for initial $\mathrm{H}_{2}$ densities of $10^{4}, 10^{5}$, and $10^{6} \mathrm{~cm}^{-3}$ and shock velocities of 20 and $40 \mathrm{~km} \mathrm{~s}^{-1}$.

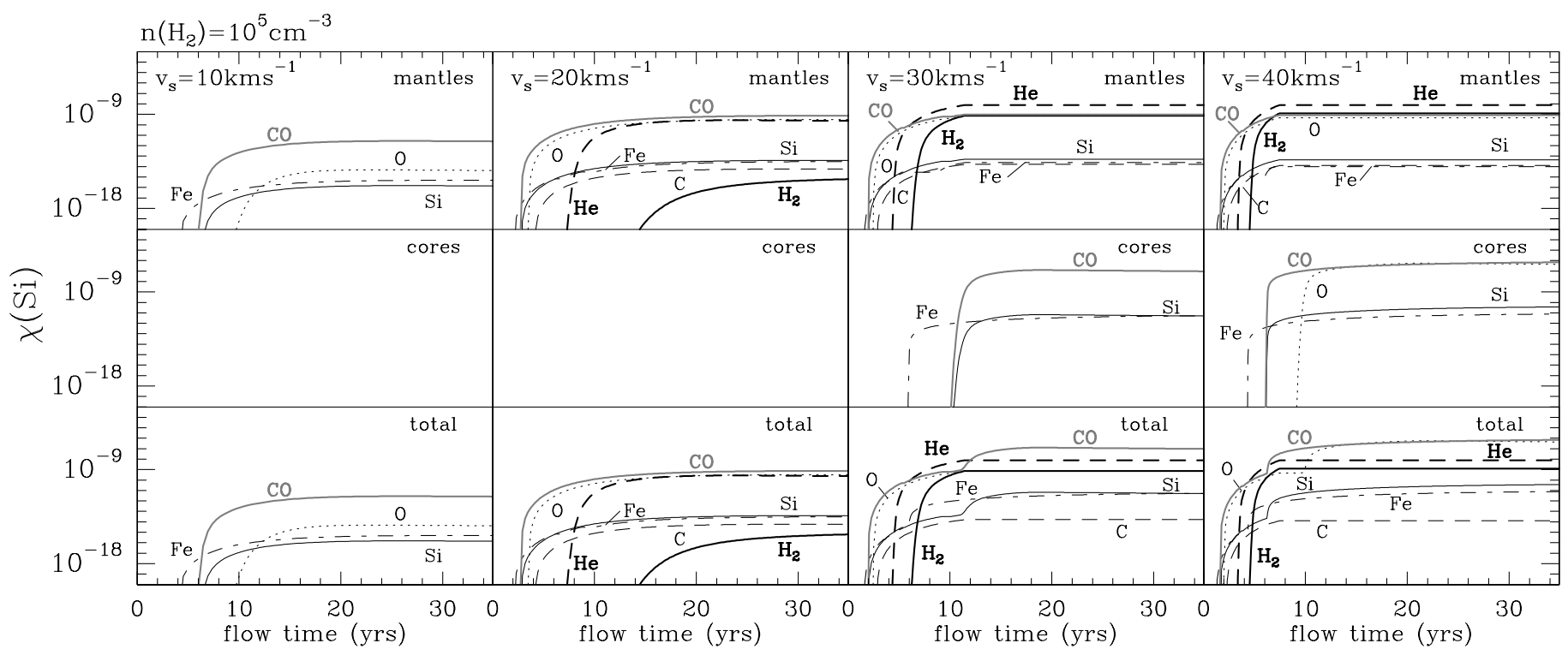

Fig. 5. Abundance of elemental silicon ejected from grains by the impact with $\mathrm{H}_{2}, \mathrm{He}, \mathrm{C}, \mathrm{O}, \mathrm{Si}, \mathrm{Fe}$, and CO, for shock velocities of 10 , 20, 30, and $40 \mathrm{~km} \mathrm{~s}^{-1}$ and a $\mathrm{H}_{2}$ gas density of $10^{5} \mathrm{~cm}^{-3}$. For each shock velocity, we show the individual production of silicon from the mantles (upper panels), from the cores (middle panels), and the total production of silicon from grains (lower panels).

lower limit of Fig. 5), and $t_{\mathrm{sat}}$ as the time for which the difference (in the logarithmic scale) of the silicon abundance between two consecutive time steps $t_{i+1}$ and $t_{\mathrm{i}}$ (see Appendix A) is $\left|\log _{10}[\chi(m)]_{i+1}-\log _{10}[\chi(m)]_{i}\right|<0.1$. Table 5 also shows the injection and saturation times caused by the contribution of all colliding particles. We note that these times are a factor of $\sim 100$ less than the typical dynamical ages of young molecular outflows like L1448-mm ( $\sim 1000 \mathrm{yr})$, but are roughly the same order of magnitude as the timescales derived for the young shocks found in this outflow ( $\leq 90 \mathrm{yr}$; Girart \& Acord 2001). From Table 5, we find that the injection and saturation times of $\mathrm{H}_{2}$ and $\mathrm{He}$ are greater than those of the heavy atoms and of $\mathrm{CO}$.

For the heavy colliding particles, $\mathrm{CO}$ seems to dominate the sputtering of the icy water mantles in low-velocity shocks. Although $\mathrm{Fe}$ initiates the grain sputtering ( $\mathrm{Fe}$ has the shortest injection and saturation times; see Table 5), its low fractional abundance prevents large enhancements of silicon by the impact with this element $\left(\leq 3 \times 10^{-14}\right.$ for $v_{\mathrm{s}} \leq 20 \mathrm{~km} \mathrm{~s}^{-1}$; Fig. 5). In contrast, collisions with $\mathrm{CO}$, whose injection and saturation times are very similar to those of $\mathrm{Si}$ but whose initial abundance is 4 orders of magnitude larger than that of $\mathrm{Si}$, produce the main injection of silicon from the mantles. The saturation times for $\mathrm{CO}$ are indeed very similar to those derived for all colliding particles at low shock velocities (see Table 5).

The abundance of silicon sputtered by collisions with $\mathrm{CO}$ for $v_{\mathrm{s}} \leq 10 \mathrm{~km} \mathrm{~s}^{-1}$ is very small $\left(\sim 10^{-12}\right.$; Fig. 5). However, at slightly higher shock velocities $\left(v_{\mathrm{s}}=20 \mathrm{~km} \mathrm{~s}^{-1}\right)$, this abundant molecule can eject from the mantles considerable amounts of this element $\left(\sim 10^{-9}\right)$. Averaging this silicon abundance over the dissipation region (shock length scale of $\sim 7 \times 10^{15} \mathrm{~cm}$; see Table 4), we estimate that the total column density of silicon injected into the gas phase in a $20 \mathrm{~km} \mathrm{~s}^{-1}$-shock is $\sim 10^{12} \mathrm{~cm}^{-2}$. While shock velocities of $v_{\mathrm{s}} \geq 25 \mathrm{~km} \mathrm{~s}^{-1}$ were required to obtain $\mathrm{Si} / \mathrm{SiO}$ column densities of $\geq 10^{12} \mathrm{~cm}^{-2}$ in May et al. (2000), we find that the inclusion of silicon as a minor constituent of the grain mantles reduces the sputtering threshold velocity by, 
Table 5. Injection and saturation times for the grain mantles and the grain cores for a medium with an initial $\mathrm{H}_{2}$ density of $n_{0}=10^{5} \mathrm{~cm}^{-3}$.

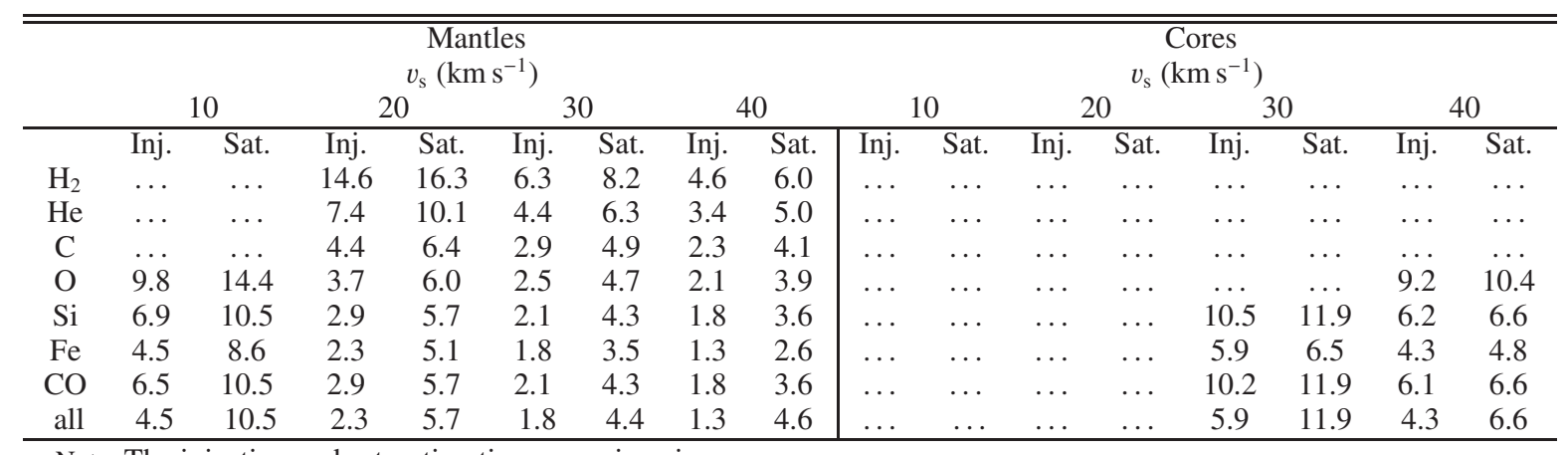

Note. The injection and saturation times are given in yr.

at least, $\left|\sim 5 \mathrm{~km} \mathrm{~s}^{-1}\right|$ in our model with respect to previous results. This velocity threshold is even reduced by $\left|\sim 10 \mathrm{~km} \mathrm{~s}^{-1}\right|$ compared to the results of Caselli et al. (1997).

It is also interesting to note that $\mathrm{CO}$ can also generate silicon abundances of $\sim 2 \times 10^{-11}$ at the very early stages of low-velocity shocks ( $t \leq 10 \mathrm{yr}$; see cases with $v_{\mathrm{s}}=20$ and $30 \mathrm{~km} \mathrm{~s}^{-1}$ in Fig. 5). As discussed in Sect. 5, these results could explain the detection of $\mathrm{SiO}$ abundances of $\sim 10^{-11}$ associated with the narrow $\mathrm{SiO}$ emission observed in the young shocks of the L1448-mm outflow.

For shocks with $v_{\mathrm{s}} \geq 30 \mathrm{~km} \mathrm{~s}^{-1}$, He plays an important role in the sputtering of the mantles. Note that the saturation times for all colliding particles at these shock velocities, deviate slightly from those of $\mathrm{CO}$ due to the increasing efficiency of He at eroding the grain mantles (Table 5 and Fig. 5). Almost all silicon within the mantles $\left(\sim 8 \times 10^{-9}\right)$ is injected into the gas phase by collisions with He for shock velocities of $v_{\mathrm{s}} \sim 30 \mathrm{~km} \mathrm{~s}^{-1}$.

For the sputtering of the grain cores, only collisions with $\mathrm{O}$, $\mathrm{Si}, \mathrm{Fe}$, and $\mathrm{CO}$ are efficient enough to destroy the cores. The injection of silicon into the gas phase from the cores occurs for shock velocities $v_{\mathrm{s}} \geq 30 \mathrm{~km} \mathrm{~s}^{-1}$, which is consistent with the results of May et al. (2000). For $v_{\mathrm{s}}=40 \mathrm{~km} \mathrm{~s}^{-1}$, only $\sim 3 \%$ of the total amount of silicon locked into the grain cores is released into the gas phase (abundance of $\sim 10^{-6}$; see Fig. 5). As for the mantles, and although $\mathrm{Si}$ has the same injection and saturation times as those of CO (both are assumed to have similar sputtering properties for the cores; see Sect. 3.2), CO is the main sputtering agent of the grain cores since its initial fractional abundance clearly exceeds that of Si.

\section{Comparison with observations: the SiO abundances}

If we now assume that silicon is rapidly oxidized into $\mathrm{SiO}$ (Pineau des Forêts et al. 1997) or that $\mathrm{SiO}$ is directly released from grains (Martín-Pintado et al. 1992), we can directly compare our predictions of the silicon abundance ejected from grains by the sputtering, with the $\mathrm{SiO}$ abundances observed in very young bipolar outflows like in L1448-mm.

In Fig. 6, we show the $\mathrm{SiO}$ abundances measured for the different velocity components detected in this outflow (ambient gas, the shock precursor component, the moderate velocity gas, and the high-velocity gas; Jiménez-Serra et al. 2005; Martín-Pintado et al. 1992) as a function of the flow time. For the ambient and precursor components, we assumed central radial velocities of $v_{\mathrm{LSR}}=4.7$ and $5.2 \mathrm{~km} \mathrm{~s}^{-1}$, respectively (Jiménez-Serra et al. 2004). Subtracting the ambient cloud

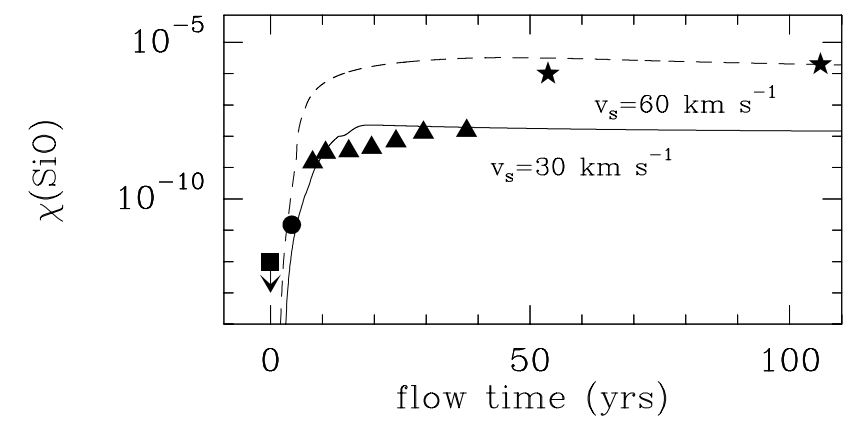

Fig. 6. Predicted $\mathrm{SiO}$ abundances within a $30 \mathrm{~km} \mathrm{~s}^{-1}$ and a $60 \mathrm{~km} \mathrm{~s}^{-1}$ shock as a function of the flow time (see shock parameters in Table 6). Observational $\mathrm{SiO}$ abundances derived in the ambient gas (filled square), the precursor component (filled circle), the moderate-velocity gas (filled triangles), and the high-velocity regime (filled stars) found in the L1448-mm outflow (Martín-Pintado et al. 1992; Jiménez-Serra et al. 2005), are also shown. The black arrow indicates an upper limit to the $\mathrm{SiO}$ abundance. The observed flow times have been derived from Eq. (2) of Sect. 2 (see text and Appendix A for details).

velocity of $v_{\mathrm{cl}}=4.7 \mathrm{~km} \mathrm{~s}^{-1}$ characteristic of the molecular emission in L1448-mm from the central radial velocities of these components (see Eq. (5) in Sect. 2), we obtain flow velocities of $v_{\mathrm{n}}=0$ and $0.5 \mathrm{~km} \mathrm{~s}^{-1}$, which correspond to flow times of $t=0$ and $4.0 \mathrm{yrs}$ for the ambient gas and the precursor component in a $30 \mathrm{~km} \mathrm{~s}^{-1}$-shock (Eq. (2) of Sect. 2; see also Eq. (A.7) in Appendix A for the details on the computation of the flow times). For the moderate velocity gas, we considered velocity intervals of $1 \mathrm{~km} \mathrm{~s}^{-1}$-width between 6 and $18 \mathrm{~km} \mathrm{~s}^{-1}$ (Jiménez-Serra et al. 2005). The SiO abundances for the ambient, precursor, and moderate velocity gas were derived assuming optically thin emission and excitation temperatures of $\sim 10-15 \mathrm{~K}$ (Requena-Torres et al. 2007). For the high-velocity gas, the $\mathrm{SiO}$ abundances of $\sim 10^{-6}$ and $2 \times 10^{-6}$ (Fig. 6) have been taken from Table 6 in Martín-Pintado et al. (1992) for the velocity ranges of $-50 \mathrm{~km} \mathrm{~s}^{-1} \leq V_{\mathrm{LSR}} \leq-40 \mathrm{~km} \mathrm{~s}^{-1}$ and $-60 \mathrm{~km} \mathrm{~s}^{-1} \leq V_{\mathrm{LSR}} \leq-50 \mathrm{~km} \mathrm{~s}^{-1}$. The flow time associated with each velocity interval is again estimated from their central radial velocities, $v_{\mathrm{LSR}}$, after subtracting the ambient cloud velocity of the L1448-mm outflow of $v_{\mathrm{cl}}=4.7 \mathrm{~km} \mathrm{~s}^{-1}$ (Eq. (5)).

Figure 6 also shows the silicon abundances, as a function of time, predicted by our model for the shocks that best fit the observational $\mathrm{SiO}$ data (with $v_{\mathrm{s}}=30 \mathrm{~km} \mathrm{~s}^{-1}$ and $v_{\mathrm{s}}=60 \mathrm{~km} \mathrm{~s}^{-1}$ ). The shock parameters used to reproduce the physical structure of these shocks are shown in Table 6. 
Table 6. Parameters of the C-shock models that best fit the $\mathrm{SiO}$ and $\mathrm{CH}_{3} \mathrm{OH}$ observational data shown in Figs. 6 and 7.

\begin{tabular}{|c|c|c|c|c|c|c|c|c|c|}
\hline $\begin{array}{c}v_{\mathrm{s}} \\
\left(\mathrm{km} \mathrm{s}^{-1}\right)\end{array}$ & $\begin{array}{l}n\left(\mathrm{H}_{2}\right) \\
\left(\mathrm{cm}^{-3}\right)\end{array}$ & $\begin{array}{c}B_{0} \\
(\mu \mathrm{G})\end{array}$ & $\begin{array}{c}\Delta \\
(\mathrm{pc})\end{array}$ & $\begin{array}{c}T_{\mathrm{n}, \max } \\
(\mathrm{K})\end{array}$ & $\begin{array}{c}z_{\mathrm{n}} \\
(\mathrm{cm})\end{array}$ & $\begin{array}{c}z_{\mathrm{i}} \\
(\mathrm{cm})\end{array}$ & $\begin{array}{c}z_{\mathrm{T}} \\
(\mathrm{cm})\end{array}$ & $\begin{array}{c}a_{\mathrm{T}} \\
\left(\mathrm{K}^{1 / 6} \mathrm{~cm}^{-1}\right)\end{array}$ & $z_{\mathrm{n}} / z_{\mathrm{i}}$ \\
\hline 30 & $10^{5}$ & 450 & 0.0036 & 2000 & $2.1 \times 10^{15}$ & $5.0 \times 10^{14}$ & $8.0 \times 10^{14}$ & $2.0 \times 10^{-15}$ & 4.2 \\
\hline 60 & $10^{5}$ & 450 & 0.0072 & 6000 & $4.1 \times 10^{15}$ & $1.0 \times 10^{15}$ & $1.1 \times 10^{15}$ & $1.8 \times 10^{-15}$ & 4.0 \\
\hline
\end{tabular}

From Fig. 6, we note that the sputtering produced by the propagation of a $30 \mathrm{~km} \mathrm{~s}^{-1}$-shock perfectly matches the evolutionary trend of $\mathrm{SiO}$ toward being enhanced from the ambient to the moderate velocity gas observed in L1448-mm (Jiménez-Serra et al. 2005). The progressive erosion of the icy mantles by the sputtering with $\mathrm{CO}$ reproduces the $\mathrm{SiO}$ abundances observed in the ambient gas $\left(\leq 10^{-12}\right)$, in the precursor component $\left(\sim 10^{-11}\right)$, and in the moderate velocity gas (from $\sim 10^{-9}$ to $\sim 10^{-8}$ ). This suggests that the puzzling narrow $\mathrm{SiO}$ line detected toward the young shocks of L1448-mm can be explained by the recent erosion of the grain mantles containing a small fraction of $\mathrm{Si} / \mathrm{SiO}$ at the early stages of low-velocity shocks.

To fit the $\mathrm{SiO}$ abundances measured in the high-velocity gas, a velocity shock with $v_{\mathrm{s}}=60 \mathrm{~km} \mathrm{~s}^{-1}$ is needed to sputter $\sim 9 \%$ of the silicon contained within the olivine cores and increase the predicted silicon abundance up to a few $10^{-6}$ (Fig. 6). This shock velocity is clearly in excess of the critical velocities of $\mathrm{C}$-shocks $\left(v_{\text {crit }} \sim 40-50 \mathrm{~km} \mathrm{~s}^{-1}\right.$; see Draine et al. 1983; Smith \& Brand 1990). Le Bourlot et al. (2002) and Cabrit et al. (2004) have recently shown that the actual shock velocity limit can be increased to $v_{\text {crit }} \sim 100 \mathrm{~km} \mathrm{~s}^{-1}$ for moderate densities and high magnetic fields. However, we cannot rule out the possibility that a J-type component would be responsible for the large $\mathrm{SiO}$ abundances observed in the high-velocity gas of L1448-mm.

Given the fact that the L1448-mm outflow shows variability in its high-velocity $\mathrm{SiO}$ emission, an alternative scenario would involve the presence of two different shocks at two different evolutionary stages that would coexist within the single-dish beam of the SiO observations (Jiménez-Serra et al. 2005).

\section{Sputtered $\mathrm{CH}_{3} \mathrm{OH}$ and $\mathrm{H}_{2} \mathrm{O}$ abundances.}

In addition to $\mathrm{SiO}, \mathrm{CH}_{3} \mathrm{OH}$ and $\mathrm{H}_{2} \mathrm{O}$ are also expected to be greatly enhanced in outflow regions (see e.g. Draine et al. 1983; Kaufman \& Neufeld 1996). In Fig. 7 (upper and middle panels), we show the predicted abundances of $\mathrm{CH}_{3} \mathrm{OH}$ and $\mathrm{H}_{2} \mathrm{O}$ as a function of the flow time, for a sample of shocks with $v_{\mathrm{s}}=10,20,30$, and $40 \mathrm{~km} \mathrm{~s}^{-1}$ (see Table 4). The impact with $\mathrm{CO}$ injects $\mathrm{CH}_{3} \mathrm{OH}$ and $\mathrm{H}_{2} \mathrm{O}$ abundances as large as $\sim 10^{-7}$ and $\sim 10^{-5}$, respectively, in low-velocity shocks (see cases with $v_{\mathrm{s}}=20 \mathrm{~km} \mathrm{~s}^{-1}$; Fig. 7). These abundances are even enhanced to up to $\sim 10^{-6}$ for $\mathrm{CH}_{3} \mathrm{OH}$, and to $\sim 10^{-4}$ for $\mathrm{H}_{2} \mathrm{O}$, in shocks with only $v_{\mathrm{s}}=30 \mathrm{~km} \mathrm{~s}^{-1}$.

We can now compare the predicted abundances of $\mathrm{CH}_{3} \mathrm{OH}$ and $\mathrm{H}_{2} \mathrm{O}$ with those observed in the young shocks of the L1448-mm outflow. Figure 7 (lower panel) shows the $\mathrm{SiO}$ and $\mathrm{CH}_{3} \mathrm{OH}$ abundances observed in the ambient gas, the precursor component and the moderate velocity gas of this outflow (Jiménez-Serra et al. 2005), as a function of the flow time. In this figure, we also show the abundances of ortho- $\mathrm{H}_{2} \mathrm{O}$ derived from the line profile of the $1_{10} \rightarrow 1_{01}$ transition measured by SWAS (Benedettini et al. 2002). Since the velocity resolution of these observations $\left(\sim 1 \mathrm{~km} \mathrm{~s}^{-1}\right.$; Benedettini et al. 2002) is lower than those of the $\mathrm{SiO}$ and $\mathrm{CH}_{3} \mathrm{OH}$ spectra $\left(\sim 0.14 \mathrm{~km} \mathrm{~s}^{-1}\right.$; see Jiménez-Serra et al. 2005), we only estimated the abundances of ortho- $\mathrm{H}_{2} \mathrm{O}$ for the moderate velocity regime (dark grey triangles in Fig. 7). As in Sect. 5, the flow times associated with these abundances were inferred by subtracting the ambient cloud velocity in L1448-mm of $v_{\mathrm{cl}}=4.7 \mathrm{~km} \mathrm{~s}^{-1}$ from the central velocities, $v_{\mathrm{LSR}}$, of the observed line profiles of these molecules (see Eq. (5)). The $\mathrm{SiO}, \mathrm{CH}_{3} \mathrm{OH}$, and $\mathrm{H}_{2} \mathrm{O}$ abundances were derived assuming optically thin emission as in Jiménez-Serra et al. (2005) for $\mathrm{SiO}$ and $\mathrm{CH}_{3} \mathrm{OH}$, and as in Neufeld et al. (2000) for $\mathrm{H}_{2} \mathrm{O}$ (Eq. (1) in this work). The abundance of $\mathrm{SiO}$ (black line), $\mathrm{CH}_{3} \mathrm{OH}$ (light grey line), and $\mathrm{H}_{2} \mathrm{O}$ (dark grey line) in gas phase generated by the sputtering of the grain mantles in the $30 \mathrm{~km} \mathrm{~s}^{-1}$ shock of Sect. 5 (see Table 6) are also shown in Fig. 7.

As for $\mathrm{SiO}$, the abundance of sputtered $\mathrm{CH}_{3} \mathrm{OH}$ agrees with what is derived in the precursor component within one order of magnitude, and with those measured in the moderate-velocity gas within a factor of $\leq 5$ (see Fig. 7). We would like to stress that we have only argued the total amount of material in the grain mantles. This implies that the progressive enhancement of the $\mathrm{SiO}$ and $\mathrm{CH}_{3} \mathrm{OH}$ abundances observed for velocities of $\leq 20 \mathrm{~km} \mathrm{~s}^{-1}$ toward L1448-mm (Jiménez-Serra et al. 2005) is naturally explained by the presence of a single shock with $v_{\mathrm{s}}=$ $30 \mathrm{~km} \mathrm{~s}^{-1}$. In the case of ortho- $\mathrm{H}_{2} \mathrm{O}$, however, the abundances of this molecule in the moderate velocity gas differ by more than a factor of 10 from those predicted by our model. This could be due either to the assumption of optically thin emission for the ortho- $\mathrm{H}_{2} \mathrm{O} 1_{10} \rightarrow 1_{01}$ transition (which could have underestimated the ortho- $\mathrm{H}_{2} \mathrm{O}$ abundances; Benedettini et al. 2002) or to a beam dilution effect. Note that the SWAS beam is $\sim 240^{\prime \prime}$, i.e., 8 times larger than the $30 \mathrm{~m}$ beam of the $\mathrm{SiO} J=2 \rightarrow 1$ observations (Jiménez-Serra et al. 2005). This leads to a corrected ortho- $\mathrm{H}_{2} \mathrm{O}$ abundance of some $\sim 10^{-4}$, which is consistent with our model predictions in Fig. 7.

\section{Conclusions}

In this work, we have presented a parametric model that mimics the steady-state profile of the physical parameters of $\mathrm{C}$-shocks. The simplicity of this model has allowed, for the first time, detailed analysis of the time evolution of the sputtering of the grain mantles and the grain cores in regions with recent outflow activity. Although this approximation does not include detailed MHD modelling, we have shown that it can be used as an efficient tool to interpret the time-dependent evolution of the abundances of typical shock tracers like $\mathrm{SiO}, \mathrm{CH}_{3} \mathrm{OH}, \mathrm{H}_{2} \mathrm{O}$, or $\mathrm{NH}_{3}$ in young molecular outflows, where transient phenomena are expected to play an important role. The assumption of steadiness of the shock can be applied to these young objects, since only the early stages of the shock evolution (characterised exclusively by the interaction of the magnetic precursor) are relevant for the dustgrain sputtering process.

To calculate the sputtering of the grain mantles and the grain cores, we assumed that silicon and methanol are minor constituents of the water mantles and that olivine is the main form of silicates within the grain cores. In spite of the low fractional abundance of heavy atoms $(\mathrm{C}, \mathrm{O}, \mathrm{Si}$, and $\mathrm{Fe}$ ) and molecules $(\mathrm{CO})$ relative to $\mathrm{H}_{2}$ or He in molecular dark clouds, these species have 


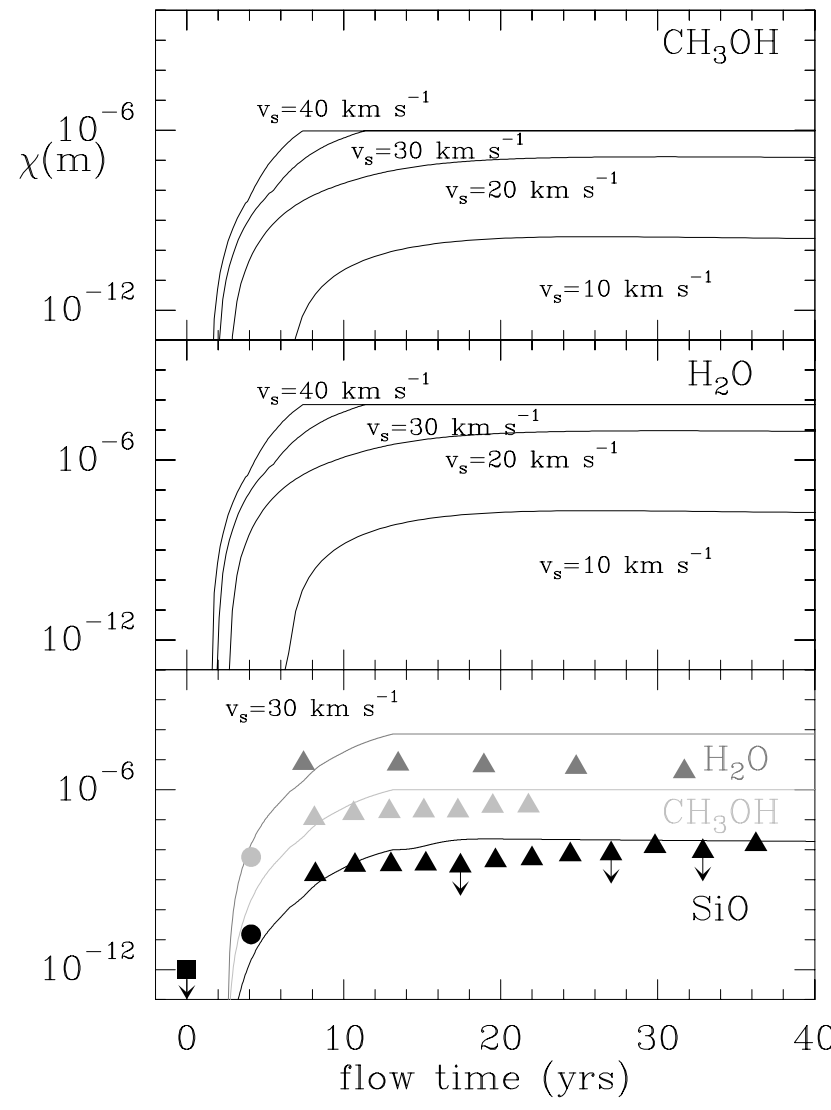

Fig. 7. Upper and middle panels: predictions of the sputtered abundances of $\mathrm{CH}_{3} \mathrm{OH}$ and $\mathrm{H}_{2} \mathrm{O}$ as a function of the flow time, for a sample of C-shocks with $v_{\mathrm{s}}=10,20,30$, and $40 \mathrm{~km} \mathrm{~s}^{-1}$ and $n_{0}=10^{5} \mathrm{~cm}^{-3}$. Lower panel: $\mathrm{SiO}, \mathrm{CH}_{3} \mathrm{OH}$, and $\mathrm{H}_{2} \mathrm{O}$ abundances ejected from the grain mantles for the $30 \mathrm{~km} \mathrm{~s}^{-1}$-shock of Table 6 as a function of time. We also show the $\mathrm{SiO}, \mathrm{CH}_{3} \mathrm{OH}$, and $\mathrm{H}_{2} \mathrm{O}$ abundances measured in the ambient gas (square), the precursor component (circles), and the moderate velocity gas (triangles) of the L1448-mm outflow (Jiménez-Serra et al. 2005; Benedettini et al. 2002). Vertical arrows indicate upper limits to the $\mathrm{SiO}$ abundance.

also been considered as sputtering agents. The relatively high abundance of $\mathrm{CO}$ with respect to the rest of heavy colliding particles and its large sputtering yield make the sputtering by $\mathrm{CO}$ very efficient. Collisions with this molecule can eject a considerably large fraction of silicon in the mantles for shocks with only $v_{\mathrm{s}} \sim 20 \mathrm{~km} \mathrm{~s}^{-1}$. This implies a reduction by $\mid \sim 5-10 \mathrm{~km} \mathrm{~s}^{-1}$ | of the threshold velocity of the sputtering with respect to other models.

By comparing the evolution of the abundances of $\mathrm{SiO}$, $\mathrm{CH}_{3} \mathrm{OH}$, and $\mathrm{H}_{2} \mathrm{O}$ predicted by our model with the abundances derived toward the different velocity regimes found in the young shocks of the L1448-mm outflow, we find that two different shocks (with $v_{\mathrm{s}}=30 \mathrm{~km} \mathrm{~s}^{-1}$ and $v_{\mathrm{s}}=60 \mathrm{~km} \mathrm{~s}^{-1}$ ) are needed to reproduce the measured abundances. The progressive enhancement of $\mathrm{SiO}$ and $\mathrm{CH}_{3} \mathrm{OH}$ observed from the ambient gas up to moderate velocities is consistent with the mantle erosion produced by a single $30 \mathrm{~km} \mathrm{~s}^{-1}$-shock. We find that the $\mathrm{SiO}$ abundance of $\sim 10^{-11}$ associated with the very narrow $\mathrm{SiO}$ emission detected in this outflow can be explained as an early product of the sputtering of the mantles by CO in low-velocity shocks if the $\mathrm{Si} / \mathrm{SiO}$ abundance in the grain mantles is $\sim 10^{-4}$ with respect to water. The disagreement between the predicted and the derived ortho- $\mathrm{H}_{2} \mathrm{O}$ abundances is probably due either to the assumption of optically thin emission for the ortho- $\mathrm{H}_{2} \mathrm{O} 1_{10} \rightarrow 1_{01}$ line observed by SWAS or to a beam dilution effect.

The approximation presented in this work will not only furnish input for more comprehensive MHD models of the shock structure in young molecular outflows, but will allow direct comparisons with the molecular line profiles observed toward these regions. These comparisons will be presented in a future paper.

Acknowledgements. We are indebted to Prof. D. R. Flower for his comments on the C-shock and sputtering theory and for kindly providing the MHD shock structure shown in Fig. 1. We acknowledge Dr. M. Kaufman for letting us use his MHD shock profile shown in Fig. 2. We would like to thank Dr. A. AsensioRamos for the help provided when developing the code, and Dr. F. Daniel for his useful comments on the manuscript. We also acknowledge an anonymous referee for helping us to significantly improve the paper. This work was supported by the Spanish MEC through projects number AYA2003-02785-E, ESP2004-00665 and ESP2007-65812-C02-01, and by the "Comunidad de Madrid" Government under PRICIT project S-0505/ESP-0277 (ASTROCAM). PC acknowledges support from the Italian Ministry of Research and University within a PRIN project.

\section{Appendix A: The C-shock structure in the shock frame}

The steady-state velocity profiles of the ion and neutral fluids, $v_{\mathrm{i}}^{*}$ and $v_{\mathrm{n}}^{*}$, within the frame of the shock, are approximated as

$v_{\mathrm{n}, \mathrm{i}}^{*}=v_{0}+\frac{\left(v_{\mathrm{s}}-v_{0}\right)}{\cosh \left[\left(z-z_{0}\right) / z_{\mathrm{n}, \mathrm{i}}\right]}$

where $z$ is the spatial coordinate and $v_{\mathrm{s}}$ the velocity of the shock. The variables $z_{0}, z_{\mathrm{i}}$, and $z_{\mathrm{n}}$ are input parameters that govern the velocity decoupling between the ions and the neutrals (see Sects. 2 and 4.1), and $v_{0}$ is required to avoid infinite compression of the far downstream gas (see Eq. (A.5) and Sect. 2). This speed only depends on the speed of the shock, $v_{\mathrm{s}}$, and the Alfvén speed, $v_{\mathrm{A}}$ (see Eq. (A.4) below). From Eq. (A.1), it is clear that the ion and neutral fluids initially move with velocities $v_{\mathrm{s}}$ and are progressively decelerated to $v_{0}$ (see Figs. 1 and 2).

Assuming that the thermal pressure of the fluid is negligible compared to the magnetic and the dynamic pressures, we can estimate the magnitude of $v_{0}$ in the downstream gas from

$\rho_{0} v_{\mathrm{s}}^{2}+\frac{B_{0}^{2}}{8 \pi}=\rho v_{0}^{2}+\frac{B^{2}}{8 \pi}$

where $\rho_{0}$ and $\rho$ are the preshock and postshock gas mass densities, and $B_{0}$ and $B$ are the magnitude of the magnetic field in the preshock and postshock regimes, respectively. Considering that the compression of the magnetic field is given by $B=B_{0}\left(v_{\mathrm{s}} / v_{0}\right)$ and that the Alfvén speed, $v_{\mathrm{A}}$, is defined as $v_{\mathrm{A}}^{2}=B_{0}^{2} /(4 \pi \rho)$, Eq. (A.2) becomes

$v_{0}^{2}=\frac{v_{\mathrm{s}}^{2}}{\rho / \rho_{0}}+\frac{v_{\mathrm{A}}^{2}}{2}\left[1-\left(\frac{\rho}{\rho_{0}}\right)^{2}\right]$.

From the principle of mass conservation, the fluid density in the postshock gas, $\rho$, is derived as $\rho=\rho_{0}\left(v_{\mathrm{s}} / v_{0}\right)$. If we now substitute the ratio $\rho / \rho_{0}$ into Eq. (A.3), we finally obtain

$v_{0}^{2}\left[v_{0}^{2}-v_{0} v_{\mathrm{s}}-\frac{v_{\mathrm{A}}^{2}}{2}\right]=-\frac{v_{\mathrm{A}}^{2}}{2} v_{\mathrm{s}}^{2}$

from which $v_{0}$ can be calculated. The particle density of the neutral fluid, $n_{\mathrm{n}}$, is given by

$n_{\mathrm{n}}=n_{0} v_{\mathrm{s}} / v_{\mathrm{n}}^{*}$ 
and the time associated with the neutral fluid or flow time, $t$, in the frame of the shock is calculated as

$t=\int \frac{\mathrm{d} z}{v_{\mathrm{n}}^{*}}$.

In each plane-parallel slab of material $i+1$ within the shock, the flow time, $t_{i+1}$, is calculated by using the trapezoidal method,

$t_{i+1}=t_{i}+\left(z_{i+1}-z_{i}\right)\left(\frac{f\left(z_{i}\right)+f\left(z_{i+1}\right)}{2}\right)$,

where $z_{i}$ and $z_{i+1}$ are the spatial coordinates for the slabs of gas $i$ and $i+1$, and $f\left(z_{i}\right)$ is defined as (see Eq. (A.6))

$f\left(z_{i}\right)=\frac{1}{v_{\mathrm{n}}^{*}\left(z_{i}\right)}$.

The temperature of the ion and neutral fluids, $T_{\mathrm{i}}$ and $T_{\mathrm{n}}$, are estimated as in Sect. 2.

\section{Appendix B: Sputtering of grains. Silicon, $\mathrm{CH}_{3} \mathrm{OH}$, and $\mathrm{H}_{2} \mathrm{O}$ fractional abundances}

The sputtering of grains has been calculated by considering different sputtering yields for the mantles and for the cores. The sputtering rate per unit volume for a spherical target of radius $a$ moving with drift velocity $v_{\mathrm{d}}$ through a Maxwellian neutral gas of temperature $T_{\mathrm{n}}$ is (Eq. (27) in Draine \& Salpeter 1979)

$$
\begin{aligned}
{\left[\frac{\mathrm{d} n(m)}{\mathrm{d} t}\right]_{\text {grain }} } & =\pi a^{2} n_{\mathrm{p}}\left(\frac{8 k T_{\mathrm{n}}}{\pi m_{\mathrm{p}}}\right)^{1 / 2} \\
& \times \frac{1}{s} \int_{x_{\mathrm{th}}}^{\infty} \mathrm{d} x x^{2} \frac{1}{2}\left[\mathrm{e}^{-(x-s)^{2}}-\mathrm{e}^{-(x+s)^{2}}\right]\langle Y(E)\rangle_{\theta}
\end{aligned}
$$

where $n_{\mathrm{p}}$ and $m_{\mathrm{p}}$ are the number density and mass of the projectile, respectively, and $s$ and $x$ are related to $v_{\mathrm{d}}, T_{\mathrm{n}}$ and the projectile impact energy $E_{\mathrm{p}}$ through $s^{2}=\frac{m_{p} v_{\mathrm{d}}^{2}}{2 k T_{\mathrm{n}}}$ and $E_{p}=x^{2} k T_{\mathrm{n}}$.

The angle-averaged sputtering yield at low energies $\langle Y(E)\rangle_{\theta}$ for the mantles can be approximated by $\langle Y(E)\rangle_{\theta} \approx 2 Y(E, \theta=0)$ (Draine 1995), where the normal-incidence yield $Y(E, \theta=0)$ is calculated as (Eq. (31); Draine \& Salpeter 1979)

$Y(E, \theta=0)=A \frac{\left(\varepsilon-\varepsilon_{0}\right)^{2}}{1+(\varepsilon / 30)^{4 / 3}}, \quad \varepsilon>\varepsilon_{0}$,

where $A$ is a constant $\left(A \approx 8.3 \times 10^{-4}\right)$, and $\varepsilon$ and $\varepsilon_{0}$ are calculated as $\varepsilon=\eta E_{\mathrm{p}} / U_{0}$ and $\varepsilon_{0}=\max [1,4 \eta]$. There, $U_{0}$ is the binding energy (per atom or molecule), and $\eta$ is derived by doing $\eta=4 \xi m_{\mathrm{p}} M_{\mathrm{t}}\left(m_{\mathrm{p}}+M_{\mathrm{t}}\right)^{-2}$, where $M_{t}$ is the target mass and $\xi$ is an efficiency factor that varies from 0.8 (for ices) to 1 (for atomic solids; Draine \& Salpeter 1979). The value of $x_{\text {th }}$, which is related to the threshold impact energy $E_{\text {th }}$, is finally calculated as $x_{\mathrm{th}}=\left(\frac{\varepsilon_{0} U_{0}}{\eta k T_{\mathrm{n}}}\right)^{1 / 2}$.

For the grain cores, we have used the sputtering yield calculated by May et al. (2000) for the impact of atomic species on olivine cores. The sputtering yield is derived from

$\langle Y(E)\rangle_{\theta}=k_{\mathrm{s}} \exp \left[-\beta /\left(E_{\mathrm{p}}-E_{\mathrm{th}}\right)\right]$

where $k_{\mathrm{s}}, \beta$, and $E_{\text {th }}$ (the sputtering threshold energy) are taken from Table 4 in May et al. (2000). In this case, $x_{\text {th }}$ is derived by doing $x_{\mathrm{th}}=\left(\frac{E_{\mathrm{th}}}{k T_{\mathrm{n}}}\right)^{1 / 2}$.
In each collision between projectile and grain, only a low fraction of silicon, $q_{\mathrm{m}}$, and $\mathrm{CH}_{3} \mathrm{OH}, r_{\mathrm{m}}$, will be ejected from the mantles $\left(q_{\mathrm{m}}=1.4 \times 10^{-4}\right.$ and $r_{\mathrm{m}}=1.4 \times 10^{-2}$; see Sect. 3.1). Analogously, only a fraction of silicon, $q_{\mathrm{c}}$, will be released from the cores $\left(q_{\mathrm{c}}=0.2\right.$ for $\mathrm{H}_{2}$ and $q_{\mathrm{c}}=1$ for the rest of colliding particles; see Sect. 3.2). If we assume a grain density $n_{\mathrm{g}}$, the total sputtering rate for $\mathrm{H}_{2} \mathrm{O}, \mathrm{CH}_{3} \mathrm{OH}$ and silicon is

$\left[\frac{\mathrm{d} n\left(\mathrm{H}_{2} \mathrm{O}\right)}{\mathrm{d} t}\right]_{\text {tot }}^{\mathrm{m}}=n_{\mathrm{g}}\left[\frac{\mathrm{d} n\left(\mathrm{H}_{2} \mathrm{O}\right)}{\mathrm{d} t}\right]_{\text {grain }}^{\mathrm{m}}$

$\left[\frac{\mathrm{d} n\left(\mathrm{CH}_{3} \mathrm{OH}\right)}{\mathrm{d} t}\right]_{\mathrm{tot}}^{\mathrm{m}}=n_{\mathrm{g}} r_{\mathrm{m}}\left[\frac{\mathrm{d} n\left(\mathrm{CH}_{3} \mathrm{OH}\right)}{\mathrm{d} t}\right]_{\text {grain }}^{\mathrm{m}}$

$\left[\frac{\mathrm{d} n(\mathrm{Si})}{\mathrm{d} t}\right]_{\mathrm{tot}}^{\mathrm{m}, \mathrm{c}}=n_{\mathrm{g}} q_{\mathrm{m}, \mathrm{c}}\left[\frac{\mathrm{d} n(\mathrm{Si})}{\mathrm{d} t}\right]_{\text {grain }}^{\mathrm{m}, \mathrm{c}}$

where $\mathrm{m}$ and $\mathrm{c}$ denotes the sputtering rate for the mantles and the cores.

By using Euler's algorithm, we calculate the total volume density of $\mathrm{H}_{2} \mathrm{O}, \mathrm{CH}_{3} \mathrm{OH}$, and $\mathrm{Si}$ ejected from grains in each plane-parallel slab of material $i$ within the shock as

$n\left(\mathrm{H}_{2} \mathrm{O}\right)_{i+1}=n\left(\mathrm{H}_{2} \mathrm{O}\right)_{i}+\Delta t\left[\frac{\mathrm{d} n\left(\mathrm{H}_{2} \mathrm{O}\right)}{\mathrm{d} t}\right]_{\mathrm{tot}, i}^{\mathrm{m}}$

$n\left(\mathrm{CH}_{3} \mathrm{OH}\right)_{i+1}=n\left(\mathrm{CH}_{3} \mathrm{OH}\right)_{i}+\Delta t\left[\frac{\mathrm{d} n\left(\mathrm{CH}_{3} \mathrm{OH}\right)}{\mathrm{d} t}\right]_{\mathrm{tot}, i}^{\mathrm{m}}$

$n(\mathrm{Si})_{i+1}=n(S i)_{i}+\Delta t\left[\frac{\mathrm{d} n(\mathrm{Si})}{\mathrm{d} t}\right]_{\mathrm{tot}, i}^{\mathrm{m}, \mathrm{c}}$

where $\Delta t=\left(t_{i+1}-t_{\mathrm{i}}\right)$. In this case, the flow time at the slab of material $i+1, t_{i+1}$, is numerically calculated as in Appendix A (see Eq. (A.7)), but using the function $f\left(z_{i}\right)$ defined as (see Sect. 2)

$f\left(z_{i}\right)=\frac{1}{v_{\mathrm{s}}-v_{\mathrm{n}}\left(z_{i}\right)}$.

The fractional abundance of silicon, $\mathrm{H}_{2} \mathrm{O}$, and $\mathrm{CH}_{3} \mathrm{OH}$ is finally derived by doing $\chi(m)=n(m) / n\left(H_{2}\right)$ in each slab of material.

\section{References}

Anders, E., \& Grevesse, N. 1989, Geochim. Cosmochim. Acta, 53, 197 Benedettini, M., Viti, S., Giannini, T., et al. 2002, A\&A, 395, 657 Cabrit, S., Flower, D. R., Pineau des Forêts, G., et al. 2004, Ap\&SS, 292, 501 Caselli, P., Hartquist, T. W., \& Havnes, O. 1997, A\&A, 322, 296

Chapman, J. F., \& Wardle, M. 2006, MNRAS, 371, 513

Chièze, J.-P., Pineau des Forêts, G., \& Flower D. R. 1998, MNRAS, 295, 672

Dopita, M. A., \& Sutherland, R. S. 2003, in Astrophysics of the Diffuse Universe (Berlin, New York: Springer), 212

Draine, B. T. 1995, Ap\&SS, 233, 111

Draine, B. T., \& Salpeter, E. E. 1979, ApJ, 231, 77

Draine, B. T., Roberge, W. G., \& Dalgarno, A. 1983, ApJ, 264, 485

Falle, S. A. E. G. 2003, MNRAS, 344, 1210

Field, D., May, P. W., Pineau des Forêts, G., \& Flower, D. R. 1997, MNRAS, 285,839

Flower, D. R., \& Pineau des Forêts, G. 2003, MNRAS, 343, 390

Flower, D. R., Pineau des Forêts, G., Field, D., \& May, P. W. 1996, MNRAS, 280,447

Girart, J. M., \& Acord, J. M. P. 2001, ApJ, 552, L63

Graedel, T. E., Langer, W. D., \& Frerking, M. A. 1982, ApJS, 48, 321 
Herbst, E., \& Leung, C. M. 1989, ApJS, 69, 271

Jiménez-Serra, I., Martín-Pintado, J., Rodríguez-Franco, A., \& Marcelino, N. 2004, ApJ, 603, L49

Jiménez-Serra, I., Martín-Pintado, J., Rodríguez-Franco, A., \& Martín, S. 2005, ApJ, 627, L121

Kaufman, M. J., \& Neufeld, D. A. 1996, ApJ, 456, 250

Le Bourlot, J., Pineau des Forêts, G., Flower, D. R., \& Cabrit, S. 2002, MNRAS, 332,985

Lesaffre, P., Chièze, J.-P., Cabrit, S., \& Pineau des Forêts, G. 2004, A\&A, 427, 147

Martín-Pintado, J., Bachiller, R., \& Fuente, A. 1992, A\&A, 254, 315

May, P. W., Pineau des Forêts, G., Flower, D. R., et al. 2000, MNRAS, 318, 809 Neufeld, D. A., Snell, R. L., Ashby, M. L. N., et al. 2000, ApJ, 539, L107

Pilipp, W., \& Hartquist, T. W. 1994, MNRAS, 267, 801

Pilipp, W., Hartquist, T. W., \& Havnes, O. 1990, MNRAS, 243, 685
Pineau des Forêts, G., Flower, D. R., \& Chièze, J.-P. 1997, in Herbig-Haro flows and the birth of stars, ed. B. Reipurth, \& C. Bertout (Kluwer Academic Publishers), IAU Symp., 182, 199

Prasad, S. S., \& Huntress, W. T. Jr. 1982, ApJ, 260, 590

Requena-Torres, M. A., Marcelino, N., Jiménez-Serra, I., et al. 2007, ApJ, 655, L37

Smith, M. D., \& Brand, P. W. J. L. 1990, MNRAS, 242, 495

Smith, M. D., Brand, P. W. J. L., \& Moorhouse, A. 1991, MNRAS, 248, 730

Snow, T. P., \& Witt, A. N. 1996, ApJ, 468, L65

Tielens, A. G. G. M., \& Allamandola, L. J. 1987, in Physical Processes in Interstellar Clouds, ed. G. E. Morfill, \& M. Scholer (Dordrecht: Reidel), 333

Tielens, A. G. G. M., McKee, C. F., Seab, C. G., \& Hollenbach, D. J. 1994, ApJ, 431,321

Whittet, D. C. B., \& Duley, W. W. 1991, A\&ARv., 2, 167

Ziurys, L. M., Friberg, P., \& Irvine, W. M. 1989, ApJ, 343, 201 